\title{
Computationally Guided Design of BCR-ABL Tyrosine Kinase Inhibitors
}

\author{
Chenghao Duan ${ }^{1}$, Simon K. S. Chu ${ }^{2}$, Justin B. Siegel ${ }^{1,3,4}$ and Jason S. Fell ${ }^{*}, 4$ \\ ${ }^{1}$ Department of Chemistry, University of California Davis, Davis, California, United States of \\ America \\ ${ }^{2}$ Biophysics Graduate Group, University of California, Davis, California, United States of America \\ ${ }^{3}$ Department of Biochemistry \& Molecular Medicine, University of California, Davis, California, \\ United States of America \\ ${ }^{4}$ Genome Center, University of California, Davis, California, United States of America
}

\begin{abstract}
BCR-ABL tyrosine kinase inhibitors (TKI) are used to treat the chronic myeloid leukemia (CML). Many TKI have been developed as the primary treatment to the CML. Imatinib, a first generation TKI, directly targets BCR-ABL with effective results. As the disease becomes more advanced, patients start to develop resistance to imatinib. Due to this effect it is necessary to generate novel treatments for advanced stage CML. Computational tools can predict new drug candidates to target BCR-ABL. We have designed two new drug candidates with different levels of modification, based on the predicted structure activity relationships with BCR-ABL. These new drug candidates are predicted to have better binding affinities with BCR-ABL than imatinib, which can be more potent treatments of the disease.
\end{abstract}

\section{INTRODUCTION}

Chronic myeloid leukemia (CML) is a fatal blood cancer with 1 to 2 cases out of 100,000 people per year and is relatively common in elderly people with a median age of diagnosis of around 65 years old. ${ }^{1,2}$ Nearly $20 \%$ to $30 \%$ of all CML patients will die within 2 years of diagnosis, and nearly $25 \%$ will die each year after that. ${ }^{3,4,5}$ The disease manifests from the fusion of chromosomes 9 and 22, creating what is known as the Philadelphia chromosome, within hematopoietic stem cells. ${ }^{1}$ From this chromosomal fusion the ABL tyrosine kinase and the BCR genes fuse together to form a unique chimeric BCR-ABL oncoprotein. Since the pathogenesis of the disease is stringently related to BCR-ABL, primary treatments have been designed to inhibit this enzyme. BCR-ABL tyrosine kinase inhibitors (TKI) are a drug family that were developed to bind to the catalytic cleft of BCR-ABL to prevent enzyme action.
The first line of treatment of CML is imatinib (Gleevec, STI-571; Scheme 1a), and most patients who are treated with imatinib in the diseases early stages achieve durable complete cytogenetic responses long-term event-free survival, and transformation-free survival. ${ }^{6}$ Before imatinib (circa 2001) CML patients were treated with combinations of chemotherapy (primarily busulfan) and interferon, which did not prolong patient life and gave an estimated 8year survival rate of nearly $6 \%$. Since 2001 , treatments with imatinib have substantially increased the 8-year survival rate of CML patients to $87 \%{ }^{7}$ Imatinib binds to the activation loop of BCR-ABL which places the enzyme in an inactive conformation. ${ }^{6}$

Imatinib contains a pyridine ring, which mimics ATP binding, and a piperazine moiety that binds hydrophobically to the BCRABL activation loop, preventing enzyme activation, this interaction is showing in figure $1 .^{8}$ 
Scheme 1. The four most common TKI: imatinib (a), nilotinib(b), dasatinib (c), and bosutinib (d).

a)<smiles>Cc1ccc(NC(=O)c2ccc(CN3CCN(C)CC3)cc2)cc1Nc1nccc(-c2cccnc2)n1</smiles>

c)<smiles>Cc1nc(Nc2ncc(C(=O)Nc3c(C)cccc3Cl)s2)cc(N2CCN(CCO)CC2)n1</smiles>

Imatinib is the first-choice therapy to treat the CML particularly in the early stages of the disease, also known as the chronic phase (CP). As patients reach the accelerated phase (AP) of CML they begin to exhibit no significant response to imatinib. ${ }^{6}$ This is due to the emergence of clones of CML with mutant forms of BCR-ABL. ${ }^{1}$ These mutations in BCR-ABL do not affect catalytic activity but decrease imatinib sensitivity. Disease resistance to imatinib has become a significant challenge in the treatment of CML.

The second-generation TKI are bosutinib (SKI-606), dasatinib (BMS-354825) and nilotinib (AMN-107), which all shows b)<smiles>Cc1cn(-c2cc(NC(=O)c3ccc(C)c(Nc4nccc(-c5cccnc5)n4)c3)cc(C(F)(F)F)c2)cn1</smiles>

d)<smiles>COc1cc(Nc2c(C#N)cnc3cc(OCCCN4CCN(C)CC4)c(OC)cc23)c(Cl)cc1Cl</smiles>

tremendous improvement in the treatment of the mutant forms of BCR-ABL (Scheme 1bd). Nilotinib is structurally similar to that of imatinib and binds BCR-ABL similarly to that of imatinib with nearly 30 times higher affinity. ${ }^{9}$ Bosutinib and dasatinib can be classified as SRC kinase inhibitors as well as a TKI. Dasatinib can bind to both the active and inactive forms of BCR-ABL nearly 325 times more than imatinib. ${ }^{10}$ Bosutinib can interact with many BCR-ABL mutants and binds nearly 200 times more than imatinib. ${ }^{10}$ While second generation TKI show promise in treating $\mathrm{CML}$ in $\mathrm{CP}$, there still is a need for more effective treatments for the AP of CML.

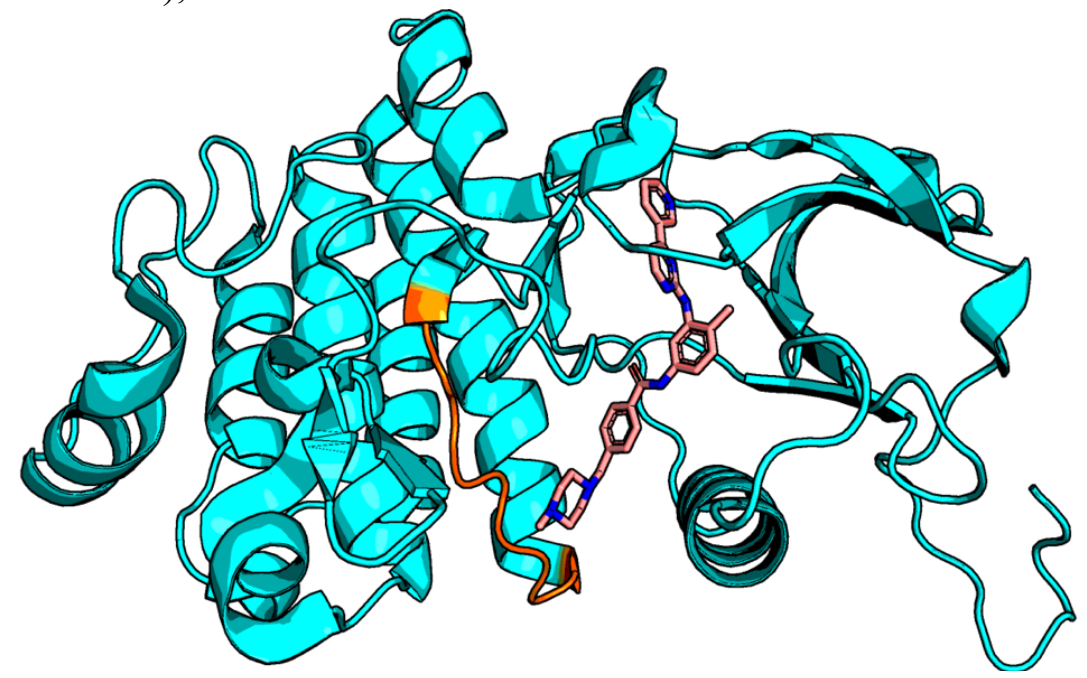

Figure 1. Imatinib (pink) within the BCR-ABL (teal) active site interacting with the activation loop (orange). 
In order to help stimulate ideas for a $3^{\text {rd }}$ generation therapeutic, in this study we have designed two new novel drug candidates derived from imatinib. We have performed computational docking of these new candidates in BCR-ABL and compared how well these two new candidates are with imatinib, dasatinib, bosutinib, and nilotinib. Our two drug candidates have computationally higher docking scores than that of imatinib, and we predict that one candidate may be an orally available treatment. These candidates have the potential to be powerful new lead TKI.

\section{METHODS}

The ATP competitive binding drug imatinib and the BCR-ABL kinase complexes were obtained from the RCSB website (1IEP). ${ }^{11}$ The crystal structure of BCR-ABL with nilotinib $(3 \mathrm{CS} 9)^{12}$, bosutinib $(4 \mathrm{MXX})^{13}$ and dasatinib (6BSD) ${ }^{14}$ inhibitors were used for computational modeling.

PyMol was used to visualize and measure interaction distances. ${ }^{15}$ ADMET properties were calculated using OpenEye FILTER, which screens drug candidates based on the bioavailability, toxicity, and the Lipinski rule of 5 (number of chiral centers, Lipinski hydrogen-bond donors, Lipinski hydrogenbond acceptors, and molecular weight). ${ }^{16}$ VBrood is used to build new drug candidates via bioisosteric replacement using imatinib as the lead molecule. ${ }^{17}$

Gaussian09 was used to optimize molecules with $\mathrm{AM} 1{ }^{18}$ semi-empirical method. ${ }^{19}$
Conformation libraries were generated using OMEGA. ${ }^{20}$ Drug candidates were docked in the active site of BCR-ABL using FRED and FRED receptor. ${ }^{21}$ FRED and FRED receptor generates a total score, steric score, protein, ligand desolvation energies, and hydrogen bond scores. The steric score measures the steric contacts of heavy atoms between the ligand and enzyme interface. The protein and ligand desolvation terms are penalties from the loss of hydrogen bonds from implicit solvent molecules with the protein and ligand, respectively, when both groups are brought together in docking. The hydrogen bonds term is calculated from hydrogen bonds formed between the ligand and enzyme. Lastly, the total score is the sum of all of the energies (steric score, protein and ligand desolvation energies, and hydrogen bond scores). These methods have been previously utilized designing new dipeptidyl peptidase4 inhibitors. $^{22}$

\section{RESULTS AND DISCUSSION}

Evaluation of existing TKI

Currently the most popular drugs for the treatment of CML are imatinib, nilotinib, dasatinib, and bosutinib. We performed docking of each of the four drug molecules into the active site of the BCR-ABL (1IEP) using FRED and FRED receptor, and the results are tabulated in Table 1. All calculated chemgauss4 and ADMET values for each drug are tabulated in the supporting information (Table S1).

Table 1. FRED receptor and ADMET values from docking imatinib, nilotinib, dasatinib, and bosutinib with BCR-ABL.

\begin{tabular}{|c|c|c|c|c|c|c|c|}
\hline & $\begin{array}{l}\text { Total } \\
\text { Score }\end{array}$ & Steric & $\begin{array}{c}\text { Protein } \\
\text { Desolvation }\end{array}$ & $\begin{array}{c}\text { Ligand } \\
\text { Desolvation }\end{array}$ & $\begin{array}{c}\text { Hydrogen } \\
\text { Bonds }\end{array}$ & $\log P$ & $\begin{array}{c}\text { Relative Binding } \\
\text { Affinity }\end{array}$ \\
\hline Imatinib & -10.47 & -14.28 & 3.74 & 4.68 & -4.62 & 2.59 & $x 1$ \\
\hline Nilotinib & -12.29 & -20.17 & 4.99 & 5.11 & -2.23 & 3.77 & $x 30$ \\
\hline Bosutinib & -9.41 & -17.00 & 4.27 & 5.04 & -1.72 & 3.64 & $x 200$ \\
\hline Dasatinib & -10.74 & -20.00 & 4.98 & 6.85 & -2.57 & 1.89 & x325 \\
\hline
\end{tabular}


Among the four of the existing BCRABL inhibitors, the order of relative drug binding affinity is imatinib, nilotinib, bosutinib, and dasatinib. The computed value that most closely resembles the relative drug binding trend is the steric score, where the values of dasatinib, nilotinib, and bosutinib $(-20.00,-20.17$, and -17.00 respectively) are more favorable than the value of imatinib (14.28). The steric score represents how well these drugs fit in the BCR-ABL active site, and FRED correctly predicts that the more pharmaceutically effective drugs fit better in the BCR-ABL active site than imatinib, however FRED predicts that dasatinib and nilotinib are nearly equal in their predicted energy. The protein and ligand desolvation penalties inversely correlate with effectiveness, that being the larger the solvation penalty the more effective the drug. The hydrogen bond score does not correlate with drug effectiveness, this suggests that hydrogen bonding is not a significant interaction between the ligand and protein. In addition, the calculated $\log \mathrm{P}(\mathrm{cLogP})$ values would suggest not all of these drug molecules are orally available, which only imatinib and dasatinib can be taken orally.

These existing treatments of CML effectively interact with BCR-ABL, however we can gain a better understanding of how these drugs interact with BCR-ABL. Figure 2 displays imatinib redocked in BCR-ABL active site (Figure 2a) with key interactions highlighted between active site residues and imatinib (Figures $2 b$ and $2 c$ ). Imatinib forms a linear shape within the active site cavity, coming into steric contact with most of the cavity residues. Hydrogen bonds are formed between residues E286, T315, and D381 with the ligand (Figure 2c). Moreover, the pyridine moiety of imatinib, which mimics ATP binding, forms a slip-stacked pi-pi interaction with F317 with distance $4.2 \AA$ (Figure 2c). We predict that these are key interactions that should be conserved in our drug designs and modifications from imatinib.

\section{Chemical Knowledge Driven Drug Design:}

We first designed a new drug candidate, Candidate 1 (Figure 3), from imatinib based on pharmaceutical chemistry knowledge and structural analysis of existing drug molecules. We started our design with reconnecting the central amide moiety to be similar to that of nilotinib. We next replaced the piperazine group with an oxazole group and moved this group from the meta position to para to the conjoining amide. By changing the piperazine group to oxazole group, the potential toxicity of the molecule should be lowered by preventing the candidate from interfering with the metabolic pathways that involving the N-dealkylation of the piperazine ring. ${ }^{23}$ The oxygen atom of the oxazole may also promote an additional hydrogen bond within the active site. We also replaced the trifluoromethyl group to a fluoro group, which reduces the inductive effect to the benzene ring and also reduces the molecular weight. After several docking pre-trials, the fluoro and oxazole groups interact with BCR-ABL best at the meta and para positions, respectively. 
a)

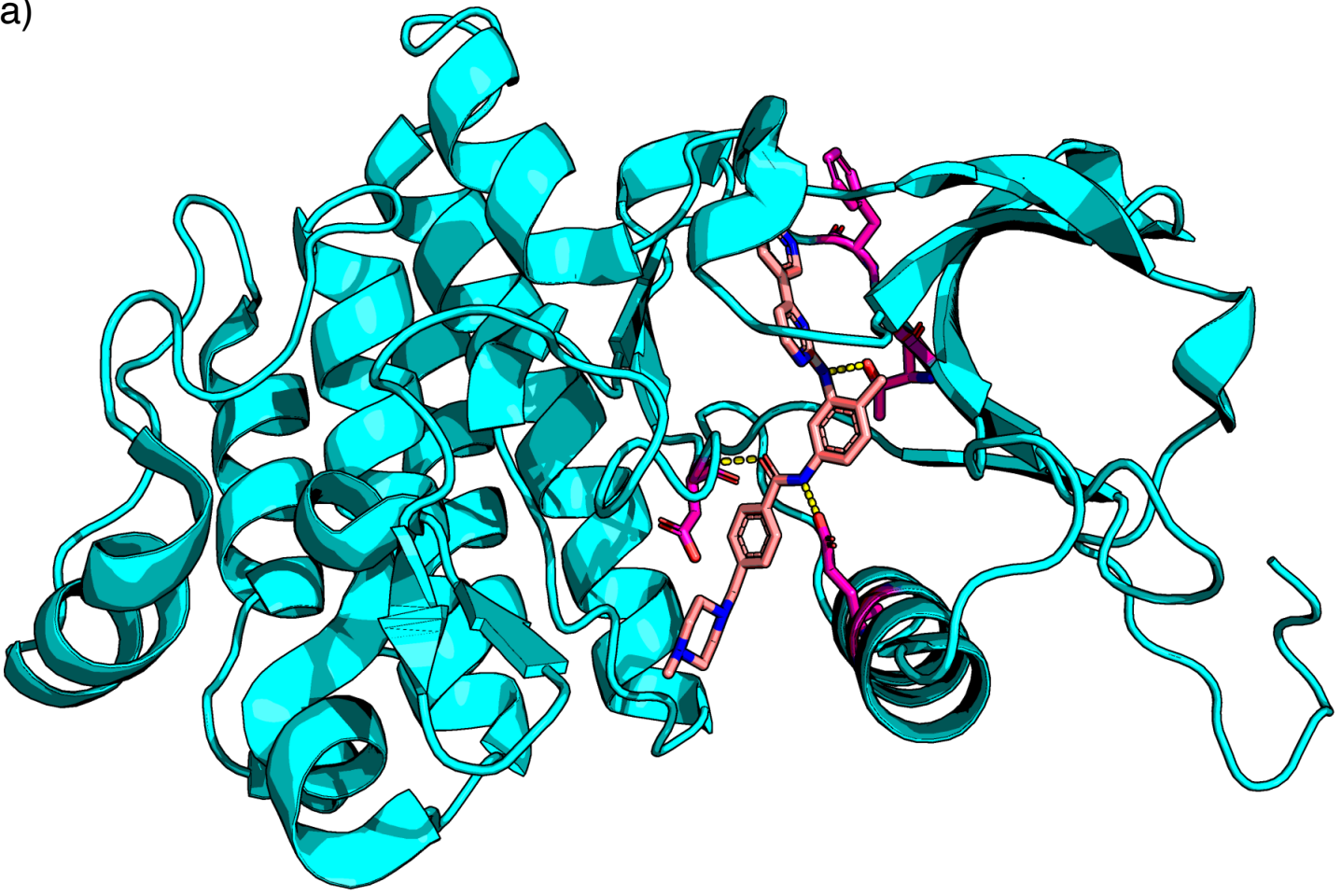

b)

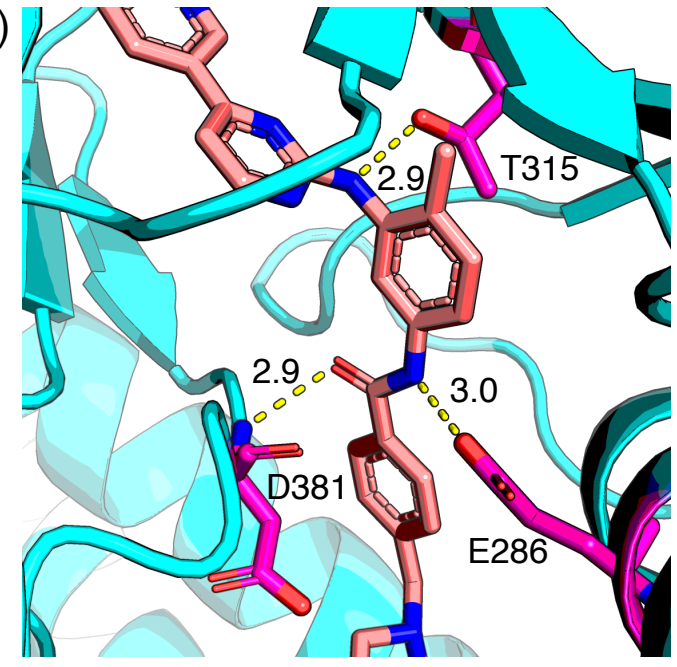

c)

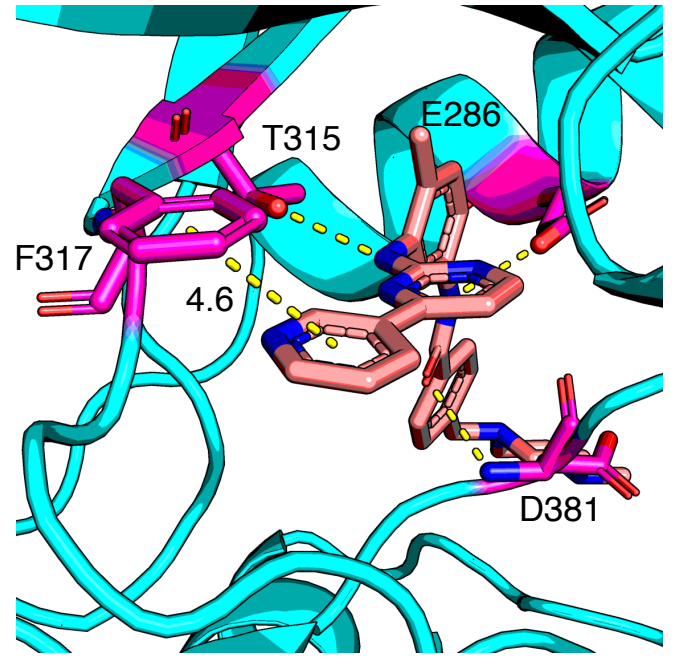

Figure 2. Imatinib (pink) redocked into BCR-ABL (teal) with interacting residues shown in purple (a). Residues E286, T315, and D381 are hydrogen bonding to imatinib (b). F317 forms a slipstacked pi-pi stacking with the pyridine moiety of imatinib (c). Distances are reported in Ångstroms $(\AA)$. 

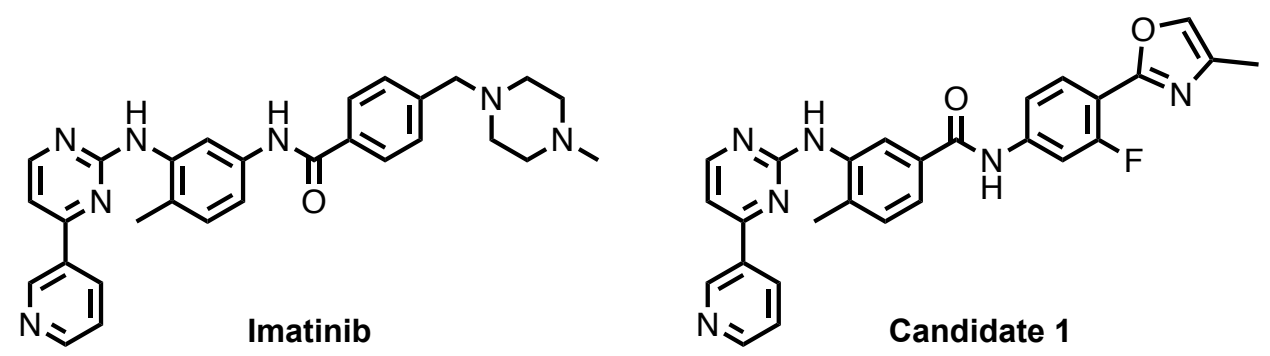

Figure 3. The structures of imatinib (left) and Candidate 1 (right).

The computed docking values of Candidate 1 with BCR-ABL are tabulated in table 2 with score comparisons to nilotinib and imatinib. Calculated chemgauss 4 and ADMET values for Candidate $\mathbf{1}$ are tabulated in the supporting information (Table S1). The docking score is more favorable for Candidate 1 than nilotinib and imatinib by 0.44 and 2.26, respectively. The steric score for Candidate 1 is lower than that of imatinib but higher than that of nilotinib, -1.26 and +4.63 respectively, which indicates that Candidate 1 may be an effective TKI in the treatment of CML. Protein and ligand desolvation penalties are also less severe for Candidate 1 than nilotinib and imatinib, and the hydrogen bond score is also more favorable. The calculated $\log \mathrm{P}$ value is similar to bosutinib and nilotinib, which we predict that Candidate 1 might be less orally available than dasatinib and imatinib.

The computed properties from docking Candidate 1 with BCR-ABL leads us to believe we potentially have a new lead molecule for future CML treatment. To better understand how this drug candidate interacts with BCR-ABL, we investigated the molecular interactions of Candidate 1 with BCR-
ABL, which is shown in Figure 4. Unlike imatinib, Candidate 1 is predicted to bend into a "U" shape within the active site cavity, which limits the steric contacts that made in the active site cavity (Figure 4a), which likely contributes to the lower steric score. The pyridine moiety of Candidate $\mathbf{1}$ is facing the "southern" side of the active site and forming a hydrogen bond with the D381 carbonyl backbone, rather than pi-pi stacking interaction with F317 (Figure 4b). The new oxazole moiety forms a new hydrogen bond with K271 (Figure 4b). Candidate 1 does not interact with E286, T315, and F317 (Figure 4c). The methyl group and the double bond contribute to the lipophilic interactions to further stabilize the structure in the active site. Candidate 1 also interacts with the activation loop, similarly to imatinib (Figure 4d).

In summary, the chemical knowledge and intuition drug designed TKI Candidate 1 displays an overall desirable result compared to nilotinib and imatinib. The total score is the lowest among all four drugs, and the steric score is lower than imatinib, which suggests Candidate 1 could be a new lead molecule.

Table 2. Computed docking values of Candidate 1 with comparisons to imatinib and nilotinib.

\begin{tabular}{|c|c|c|c|c|c|c|}
\hline & $\begin{array}{l}\text { Total } \\
\text { Score }\end{array}$ & Steric & $\begin{array}{c}\text { Protein } \\
\text { Desolvation }\end{array}$ & $\begin{array}{c}\text { Ligand } \\
\text { Desolvation }\end{array}$ & $\begin{array}{l}\text { Hydrogen } \\
\text { Bonds }\end{array}$ & $\log P$ \\
\hline Candidate 1 & -12.73 & -15.54 & 3.02 & 4.76 & -4.96 & 3.50 \\
\hline$\Delta$ Nilotinib & -0.44 & +4.63 & -1.97 & -0.59 & -2.73 & -0.27 \\
\hline$\Delta$ matinib & -2.26 & -1.26 & -0.72 & -0.08 & -0.34 & +0.91 \\
\hline
\end{tabular}


a)

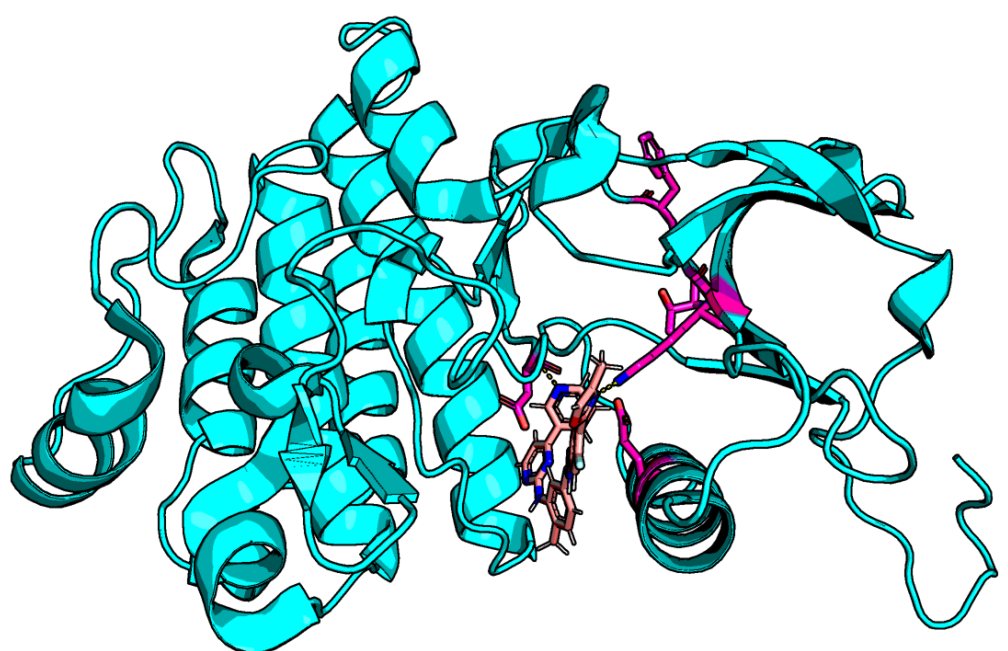

b)
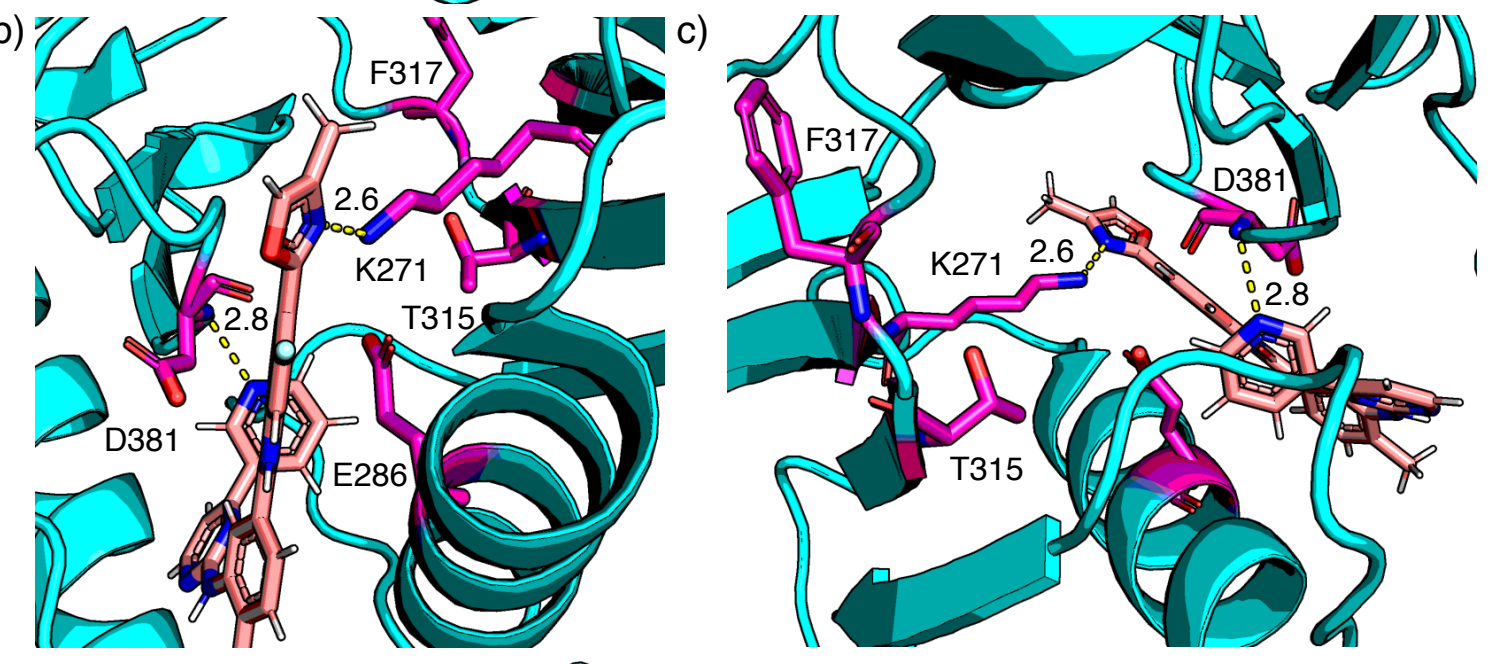

d)

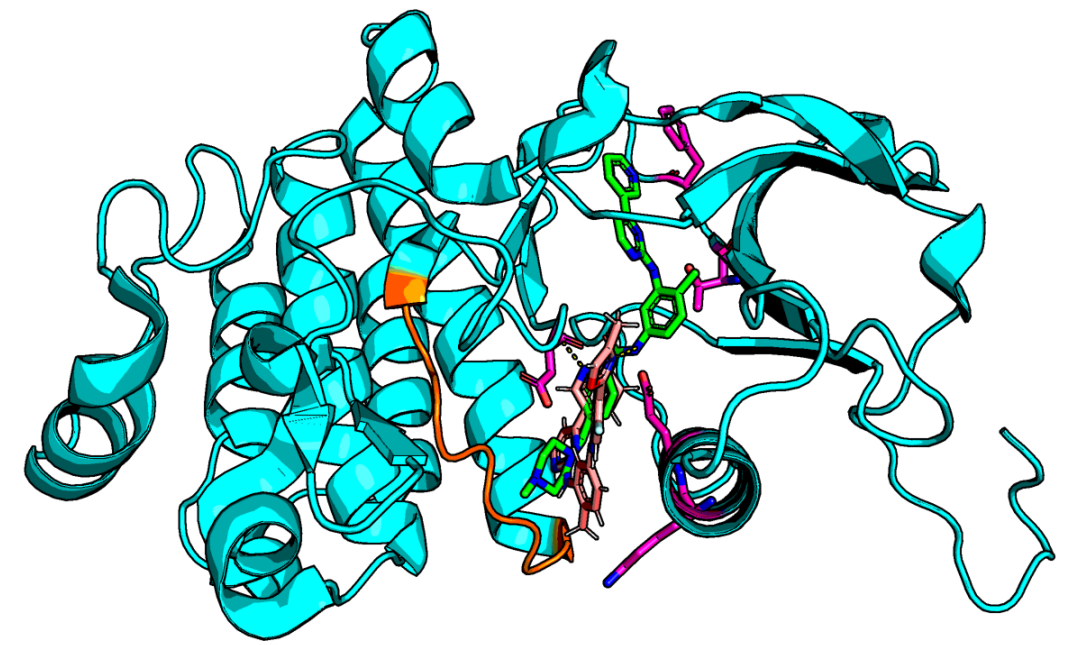

Figure 4. Candidate 1 (pink) docked into BCR-ABL (teal) with interacting residues shown in purple (a). Residues K271 and D381 are hydrogen bonding to Candidate 1 (b \& c). Overlay of Candidate 1 (pink) and imatinib (green) docked into BCR-ABL and interacting with the activation loop (orange) (d). Distances are reported in Ångstroms $(\AA)$. 

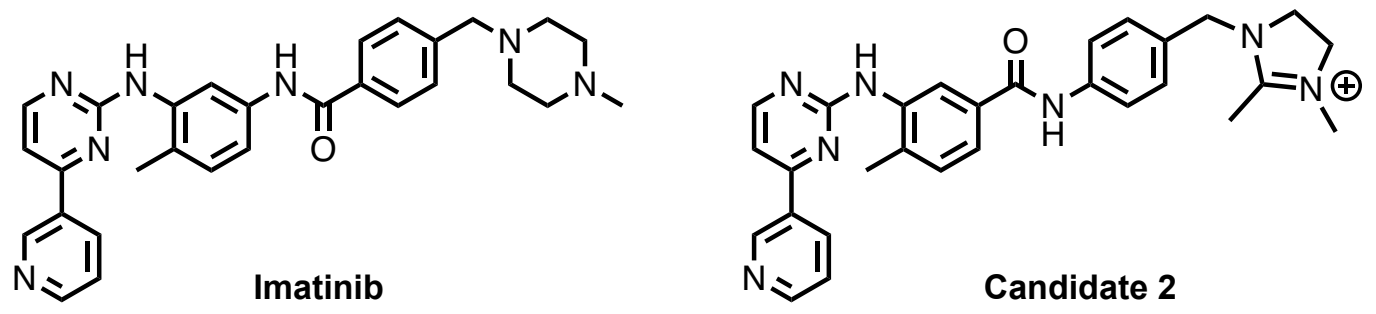

Figure 5. The structures of imatinib (left) and Candidate 2 (right).

\section{Computationally Driven Drug Design:}

While Candidate 1 was designed starting from imatinib using chemical knowledge and intuition, we also explored the design of a new drug candidate (Candidate 2) using the computational software VBrood ${ }^{17}$ (Figure 5). VBrood identified that the piperazine moiety to be replaced by a cationic $n$-methyldihydro-imidzole and that the central amide be reconnected similar to that of nilotinib.

The docking results for Candidate $\mathbf{2}$ are listed in table 3. Calculated chemgauss 4 and ADMET values for Candidate $\mathbf{2}$ are tabulated in the supporting information (Table S1). Candidate 2 has the lowest total score of -13.95 among all of the drugs and Candidate 1. The steric score for Candidate 2 is lower than imatinib and Candidate 1 but higher than nilotinib, suggesting that this candidate might be a promising new lead molecule. The hydrogen bond score is also lower than imatinib, nilotinib, and Candidate 1 . The $\log P$ value of Candidate 2 is lower than nilotinib and slightly higher than imatinib, suggesting that this drug candidate is likely equally as orally available as dasatinib and imatinib. Based on all of the computed scores we predict that the computationally designed drug (Candidate 2) would perform the best compared to the chemical knowledge-based designed drug (Candidate 1) and the four common treatments.

The key interactions between residues in the active site and Candidate $\mathbf{2}$ are displayed in Figure 6. Similar to imatinib, Candidate 2 forms a linear shape within the active site cavity. Yet the pyridine moiety faces the "southern" side of the cavity similar to that of Candidate 1 (Figure 6a). Candidate 2 also hydrogen bonds to D381 and E286, and K285 hydrogen bonds to pyridine moiety (Figure $6 b)$. The hydrogen bond to D381 is shorter by $0.4 \AA$ respective to imatinib. The cationic imidazole moiety is within the region of T315 and F317. However, this group does not interact with these residues (Figure 6c). Candidate 2 also interacts with the activation loop, similarly to imatinib (Figure 6d).

Table 3. Computed docking values of Candidate 2 with comparisons to imatinib and nilotinib.

\begin{tabular}{ccccccc} 
& Total & & Protein & Ligand & Hydrogen \\
& Score & Steric & Desolvation & Desolvation & Bonds & LogP \\
\hline Candidate 2 & -13.95 & -16.93 & 4.40 & 4.25 & -5.68 & 2.68 \\
\hline$\Delta$ Nilotinib & -1.66 & +3.24 & -0.59 & -0.86 & -3.45 & -1.09 \\
\hline$\Delta$ matinib & -3.48 & -2.65 & +0.66 & -0.43 & -1.06 & +0.09 \\
\hline
\end{tabular}


a)

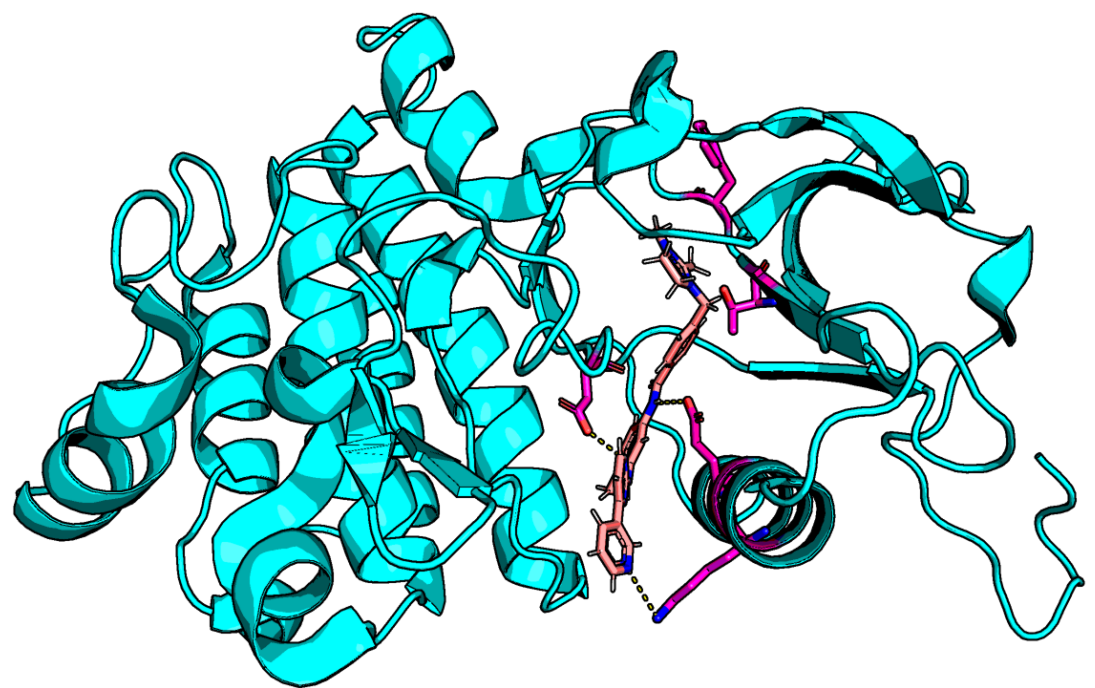

b)

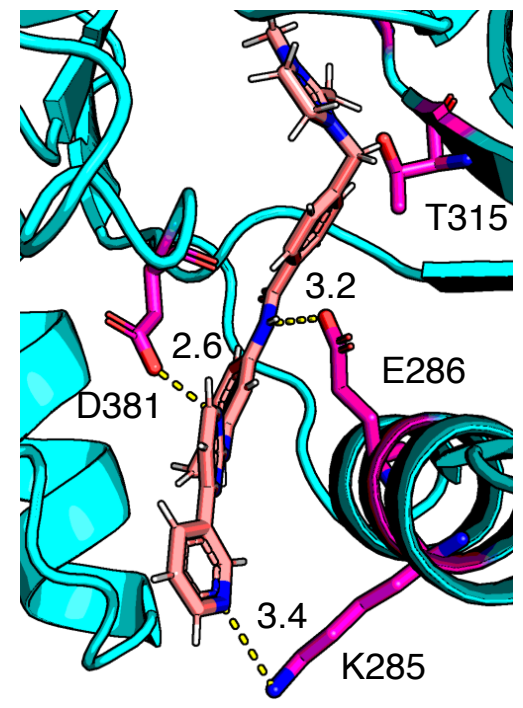

c)

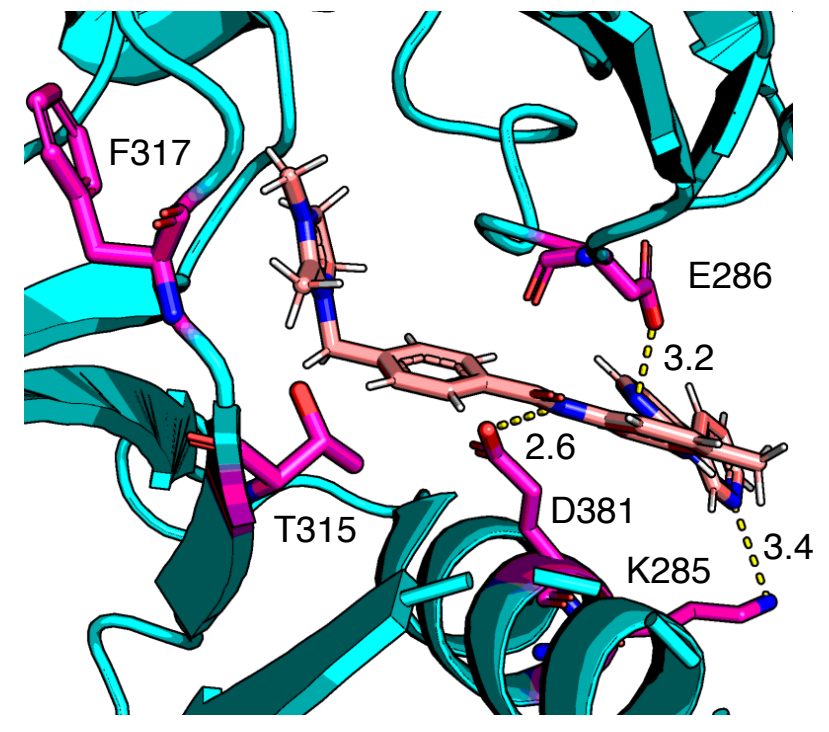

d)

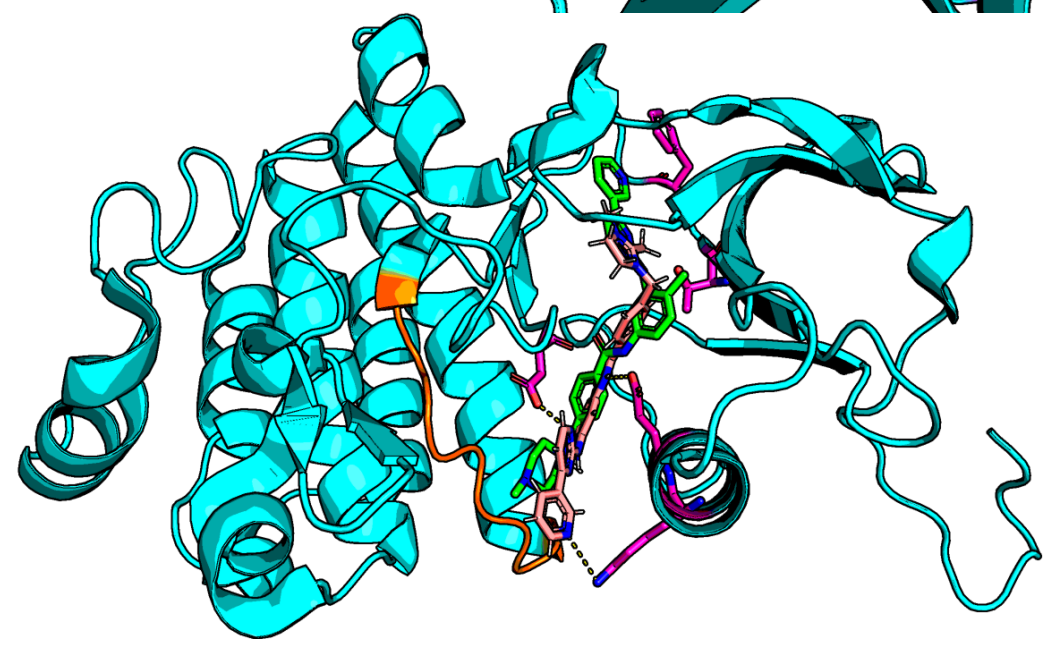

Figure 6. Candidate 2 (pink) docked into BCR-ABL (teal) with interacting residues shown in purple (a). Residues K285, E286, and D381 are hydrogen bonding to Candidate 1 (b \& c). Overlay of Candidate 2 (pink) and imatinib (green) docked into BCR-ABL and interacting with the activation loop (orange) (d). Distances are reported in Ångstroms $(\AA)$. 


\section{CONCLUSION}

As CML becomes resistant to the first line treatment imatinib, second generation drugs, nilotinib, dasatinib, and bosutinib, are utilized to inhibit BCR-ABL as the disease progresses to the CP. New TKI are needed in order to bypass drug-resistant mutants of BCR-ABL. ${ }^{24}$ We generated two new drug candidates by performing modifications on imatinib using chemical knowledge and intuition (Candidate 1), and using computational software for bioisosteres replacement (Candidate 2). Both of the drug candidates generated are predicted to be more effective than imatinib as well as potentially nilotinib. Even though these drug leads are predicted to be more advanced treatments to CML, further testing and studies in vivo are required to determine true efficacy. Despite the further studies that need to be conducted in the future, the usage of computational software and chemical knowledge serve as a good starting point to propose new drug molecules for advanced treatments. ${ }^{25}$

\section{AUTHOR INFORMATION Corresponding Author \\ *jsfell@ucdavis.edu \\ ORCID}

Jason S. Fell: 0000-0001-6680-2936

Justin B. Siegel: 0000-0003-1782-4076

Notes

The authors declare no competing financial interest.

\section{REFERENCES:}

1. Manley, P. W.; Cowan-Jacob, S. W.; Mestan, J. Advances in the Structural Biology, Design and Clinical Development of Bcr-Abl Kinase Inhibitors for the Treatment of Chronic MyeloidLeukaemia. Biochimica et Biophysica Acta (BBA) - Proteins and Proteomics 2005, 1754 (1-2), 313.

2. Hehlmann, R.; Hochhaus, A.; and Baccarani, M. Chronic myeloid leukaemia.
Science Direct, The Lancet 2007, 370, 342-250.

3. Gottschalk, S., Anderson, N., Hainz, C., Eckhardt, S. G., and Serkova, N. J. Imatinib (STI571)-Mediated Changes in Glucose Metabolism in Human Leukemia BCR-ABL-Positive Cells. Clinical Cancer Research 2004, 10, 6661-6668.

4. O'Bryen SG, Guilhot F, Larson RA, et al. Imatinib compared with interferon and low-dose cytarabine for newly diagnosed chronic-phase chronic myeloid leukemia. N Engl J Med 2003; 348:994-1004.

5. Peggs K, Mackinnon S. Imatinib mesylate: the new gold standard for treatment of chronic myeloid leukemia. $N$ Engl J Med 2003;348: 1048-50.

6. Tauchi, T., Ohyashiki, K. The Second Generation of BCR-ABL Tyrosine Kinase Inhibitors. Int J Hematol. 2006, 83

(4),294-300

7. Kantarjian H, O'Brien S, Jabbour E, et al. Improved survival in chronic myeloid leukemia since the introduction of imatinib therapy: a single-institution historical experience. Blood. 2012;119(9):1981-1987. doi:10.1182/blood-2011-08-358135

8. Nagar, B., Bornmann, W., Pellicena, P., Schindler, T., Veach, D., Miller, W., Clarkson, B., and Kuriyan, J. Crystal Structure of the c-Abl Kinase domain in complex with PD173955 and Imatinib (STI-571). Cancer Research. 2002, 62, 4236-4243.

9. Breccia, M.; Alimena, G. (2010). "Nilotinib: a second-generation tyrosine kinase inhibitor for chronic myeloid leukemia". Leukemia Research. 2009.34 (2): 129-134. doi:10.1016/j.leukres. 2009.08.031. PMID 19783301. 10. Jabbour, E., Cortes, J., and Kantarjian, H. Treatment selection after imatinib resistance in chronic myeloid leukemia. Targeted Oncology 2009, 4, 3-10.

11. RCSB PDB-1IEP: Crystal structures of the kinase domain of c-Abl in complex 
with the small molecule inhibitors

PD173955 and imatinib (STI-571).

12. RCSB PDB-3CS9: Characterization of AMN107, a selective inhibitor of native and mutant Bcr-Abl.

https://www.rcsb.org/structure/3CS9 (accessed, March $\left.3^{\text {rd }}, 2020\right)$.

13. RCSB PDB-4MXX: A conserved water-mediated hydrogen bond network defines bosutinib's kinase selectivity. http://www.rcsb.org/structure/4MXX (accessed, March $3^{\text {rd }}, 2020$ ).

14. RCSB PDB-6BSD: What Makes a Kinase Promiscuous for Inhibitors?

http://www.rcsb.org/structure/6BSD (accessed, March $\left.3^{\text {rd }}, 2020\right)$.

15. The PyMOL Molecular Graphics System; Schrödinger, LLC: 2000.

16. FILTER; OpenEye Scientific Software: Santa Fe, NM.

17. BROOD, 3.1.1.2; OpenEye Scientific Software:Santa Fe, NM

18. Dewar, M. J. S.; Zoebisch, E. G.; Healy, E. F.; Stewart, J. J. P. AM1: A New General Purpose Quantum Mechanical Molecular Model. J. Am. Chem. Soc., 1985, 107, 3902-3909.

19. Gaussian 09; Gaussian, Inc.: Wallingford,CT, 2009.

20. Hawkins, P. C. D.; Skillman, A. G.; Warren, G. L.; Ellingson, B. A.; Stahl, M. T. J. Chem Inf. Model. 2010, 50, 4, 572584.
https://www.rcsb.org/structure/1IEP (accessed, March 3rd, 2020).

21. McGann, M. J. Chem. Inf. Model. 2011, 51, 3, 578-596.

22. Tantillo, D., Siegel, J., Saunders, C., Palazzo, T., Painter, P., O’Brien, T., Nuñez, N., Nouri, D., Lodewyk, M., Hudson, B., Hare, S. and Davis, R. ComputerAided Drug Design for Undergraduates. Journal of Chemical Education. 2019, 96(5), 920-925.

23. Stepan, A. F., Walker, D. P., Bauman, J., Price, D. A., Baillie, T. A., Kalgutkar, A. S., and Aleo, M. D. Structural Alert/Reactive Metabolite Concept as Applied in Medicinal Chemistry to Mitigate the Risk of Idiosyncratic Drug Toxicity: A Perspective Based on the Critical Examination of Trends in the Top 200 Drugs Marketed in the United States. Chemical Research in Toxicology. 2011, 24, 1345-1410. 24. Weisberg, E., Manley, P., Cowan-Jacob, S. et al. Second generation inhibitors of BCR-ABL for the treatment of imatinib-resistant chronic myeloid leukaemia. Nat Rev Cancer, 2007, 7, 345-356 25. Reynolds, L. C.; Connolly, M. P.; Siegel, J. B. Computationally Guided Design of Dipeptidyl Peptidase-4 Inhibitors. BioRxiv, 772137; doi: https://doi.org/10.1101/77213 